\title{
THE CLOUDSAT EDUCATION NETWORK: SCIENTIFICALLY SIGNIFICANT COLLABORATIVE RESEARCH BETWEEN STUDENTS AND SCIENTISTS
}

\author{
Matt Rogers, Deborah Vane \\ Colorado State University, Jet Propulsion Laboratory
}

\section{INTRODUCTION}

The CloudSat Education Network (CEN) is the primary education and public outreach component of the CloudSat mission [1]. Approximately 116 schools in 16 countries around the world participate in the CEN, and are recruited from schools in the GLOBE program. Students and teachers in the CEN make atmospheric observations of temperature, precipitation, and crucially, of cloud type and cloud cover amount (including photographs of cloud observations), using a modified GLOBE Atmosphere protocol as a guide for observations. CEN observations are taken coincident with CloudSat overpasses, providing coincident spaceborne- and student surface observations. This puts students and teachers participating in the CEN at the forefront of scientific research as directly contributing partners in a collaborative research endeavor.

CEN participants make extensive use of the CloudSat/CEN webpage, which is the primary data entry portal for the CEN. Data collected from CEN students is analyzed by CloudSat scientists for quality control purposes, as well as for use in CloudSat-related research. The webpage also provides students with CloudSat overpass dates and times, as well as CEN-specific updates, and articles about CEN projects distributed through a quarterly newsletter. Besides the newsletter, active CEN schools receive periodic visits from CloudSat scientists, providing an opportunity for students and teachers to interact directly with the scientific community.

\section{STUDENT-SCIENTIST RESEARCH}

Additionally, data collected by CEN students are available as a ground-truth mechanism to analyze operational CloudSat products. A preliminary comparison study [2] used CEN-collected observations of cloud type from sixteen CEN schools during the period from 2007-2008 and 
compared the observed cloud types to those retrieved using the CloudSat 2B-CLDCLASS product [3]. In this preliminary study, there were 227 coincidental measurements between CEN schools and CloudSat overpasses, with an agreement rate of approximately $66 \%$ between the surface observers and satellite observations. A similar study, [4] conducted using WMO-trained surface observers, yielded an agreement rate of $\sim 64 \%$ between the surface observers and the satellite. Based the on results of the CEN study, a more in-depth comparison study utilizing more schools in the CEN is underway, with the goal of publishing in a peer-reviewed journal with students and scientists as co-authors.

\section{BIBLIOGRAPHY}

[1] G.L. Stephens, D.G. Vane, R.J. Boain, G.G. Mace, K.Sassen, Z. Wang, A.J. Illingworth, E.J. O'Connor, W.B. Rossow, S.L. Durden, S.D. Miller, R.T. Austin, A. Benedetti, C. Mitrescu, and the CloudSat Science Team, "The CloudSat Mission and the A-Train: A New Dimension of Space-Based Observations of Clouds and Precipitation," Bull. Amer. Meteor. Soc., 83, 17711790., 2002

[2] M. Rogers and D. Vane, "Comparison of 2B-CLDCLASS Observations to CEN-Trained Student Surface Observations," Presented at 2008 AGU Fall Meeting, San Francisco, CA, Paper A11D-0168, 2008

[3] K. Sassen and Z. Wang, "Classifying Clouds Around the Globe with the CloudSat Radar: 1Year of Results”, Geophys. Res. Lett., VOL. 35, L04805, doi:10.1029/2007GL032591, 2008

[4] Z. Wang and K. Sassen, "Cloud type and macrophysical property retrieval using multiple remote sensors," J. Appl. Meteor., 40, 1665-1682, 2001 